ISBN 978-93-86878-17-5

\title{
Generating Designs: From Crowdsourcing to Deep Learning
}

\author{
Imdat As \\ University of Hartford, West Hartford, USA
}

\begin{abstract}
In this paper, I present crowdsourcing as an alternative method to traditional methods of design acquisition. I demonstrate a case study, the Takanawa house façade project in Tokyo, which was administered at Arcbazar.com, a crowdsourcing platform for architectural design projects. I argue that crowdsourcing models can create a level playing field for designers and democratize the design space by utilizing the fair competition protocol. In this study, I demonstrate the crowdsourcing model, illustrate the results of the case study, and elaborate on future opportunities of using machine learning as a next step in generating conceptual designs.
\end{abstract}

Keywords: design delivery, architecture, crowdsourcing, deep learning, neural networks.

\section{Introduction}

Every year, there are about 15 Million design projects in the US alone, but only $11 \%$ of these projects take advantage of design professionals. [1] The customer pain is deep: Design is risky, in other words, at the end of the design process, the client may or may not like the designs generated. Moreover, locally there might be not many designers available who are interested in the project to begin with. Arcbazar.com (the platform), an online crowdsourcing platform, offers one potential answer to these pain points. It allows clients to launch design competitions, reduces their costs, tabs into a global network of designers and gives them control over the design process.

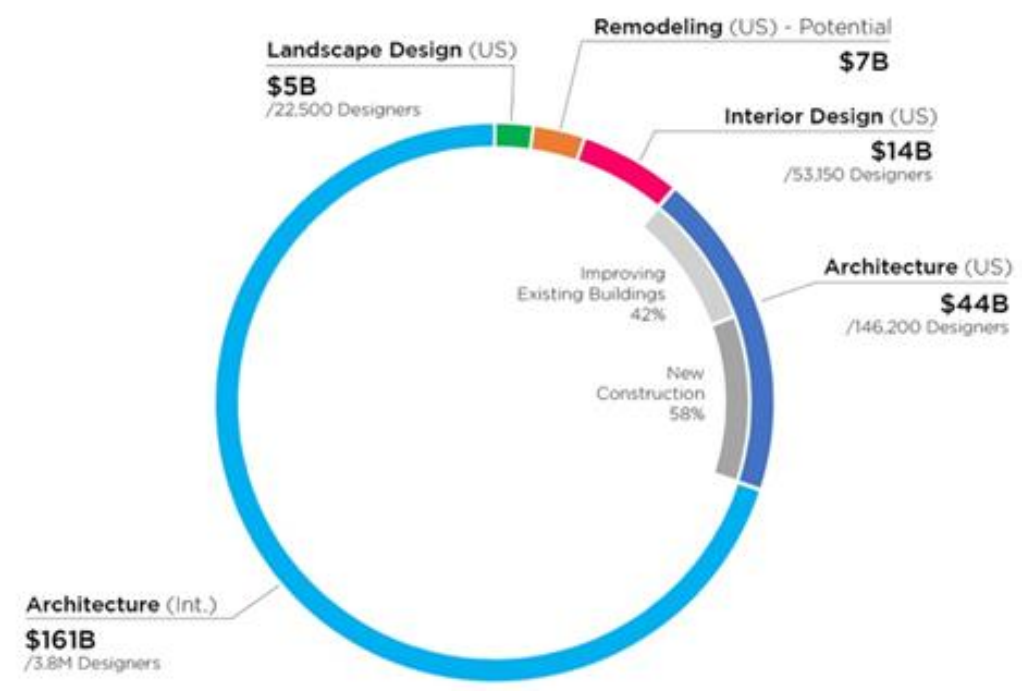

Fig. 1: Architectural Design Market Segmentation 
The design market is large: Just in the US, about \$44B is spent on architectural fees annually, \$14B on interior design fees, $\$ 7 \mathrm{~B}$ on remodeling design fees, and \$5B on landscape design fees. Internationally, $\$ 161 \mathrm{~B}$ is spent each year on design services, and there are about four million design professionals worldwide (Fig. 1). [2]

In this paper, I will present how crowdsourcing works. I will show a case study, where the platform generated designs for a façade project in Tokyo, and illustrate the methods, techniques and outcome of this project. Furthermore, I will elaborate on how crowdsourcing can potentially make use of deep learning scenarios to generate new and unprecedented designs.

\section{Background}

There are various design delivery models in architecture: a. the direct client-architect relationship, b. the request for proposals (RFP) model, c. the competition model, and d. crowdsourcing. In the direct client-architect model, the client simply finds an architect and commissions the job. In the RFP model the client interviews multiple designers, reviews their portfolios and qualifications, and commissions the job to one of them. In the competition model the client employs a competition agency which carries out a competition for the particular design challenge. In this scenario the agency develops the brief, forms the jury and delivers a winning scheme to the client. The crowdsourcing model is a variation of the competition model. However, it has no intermediary agency. Clients launch the project, decide on the timeline, the award amount and the winning scheme themselves. In other words, the client carries the full burden of the activity, but hence is in full control over the entire process.

At the platform, clients can post their design challenges and receive multiple design solutions to their design problem. It is a two-sided marketplace: Clients are on the demand-side and designers on the supply-side. Currently, $83 \%$ of clients are from the US, followed by 6\% from Europe, 5\% from Asia, and 3\% from Australia. Active designers, on the other hand, are distributed more evenly: 36\% are from the US, $43 \%$ from Europe, $15 \%$ from Asia, and 5\% from Africa (Fig.2).

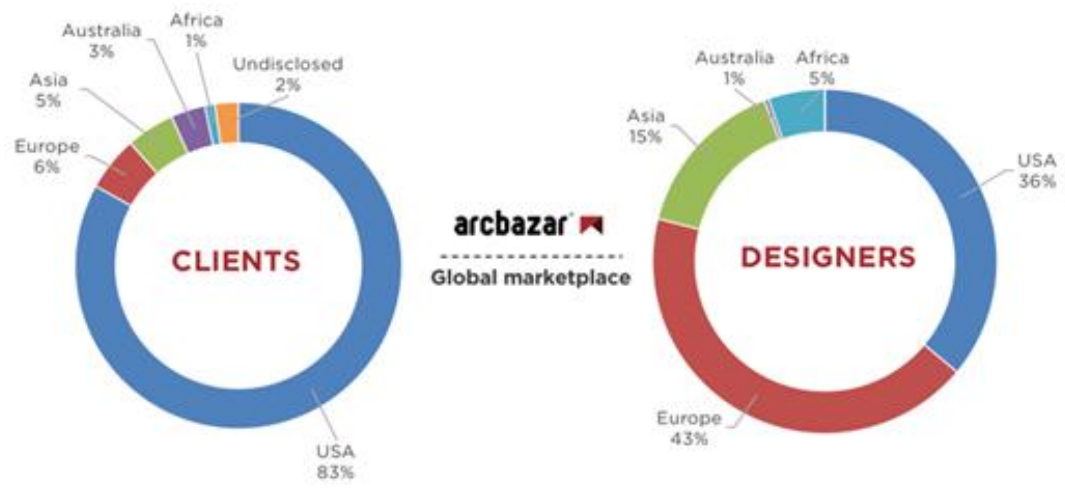

Fig. 2: Two-sided Global Marketplace

The platform completed thousands of design competitions worldwide. As of today, the distribution of project types is as follows: $44 \%$ are smaller scale remodeling projects, e.g. kitchen, bathroom, bedroom, attic etc. design challenges, $16 \%$ are interior design projects, $14 \%$ are landscape design projects, $13 \%$ are new residential projects, i.e. homes designed from scratch, $11 \%$ are commercial projects, e.g. storefronts, clinics, office spaces, etc., and about $2 \%$ are institutional projects, e.g. libraries, schools, etc. (Fig.3) 


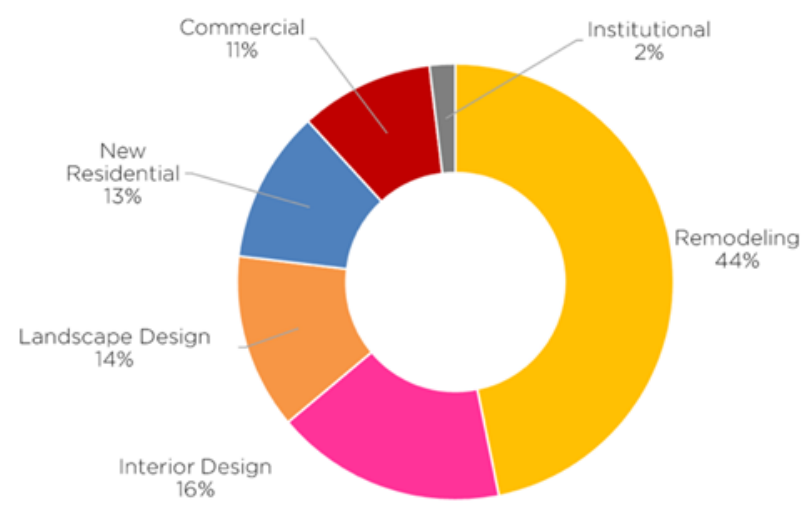

Fig. 3: Distribution of project types ran on Arcbazar.com

Clients awarded an average of $\$ 950$ to designers and received about 11 submissions per project. Each submission has about a dozen sheets, e.g. plans, sections, elevations, perspective views, etc. Thus, a client receives on average 130 sheets of documents to review. The value proposition is high, not only do clients set their own budget, but they receive a multitude of design options.

The system measures the satisfaction rate of clients with the crowdsourced end products - qualitatively and quantitatively. After clients submit their rankings the platform asks them the following questions to rate on a 1-5 scale ( 1 being worst and 5 best):

- What was the quality of the design submissions?

- Was the website easy to use?

- Was it worth your money?

- How was the communication during the competition process?

- Would you recommend the platform to your family and friends?

Quantitatively, the average is currently at a 4.3 (Fig.4). In addition, qualitatively, clients write scored reports on third party webpages, such as Angie's List, where $66 \%$ of reports were A grades, and 12\% B (based on 234 client reports). Both facts, illustrate the satisfaction rate of clients with the crowdsourcing design delivery model.

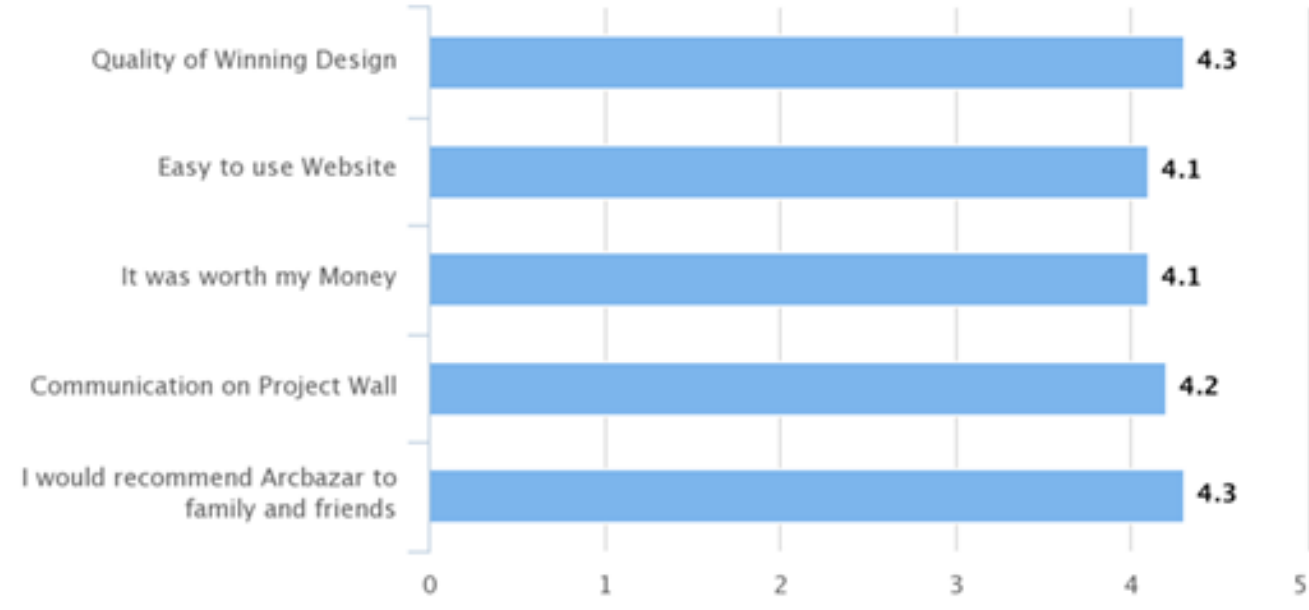

Fig. 4: Quantitative satisfaction score of clients 


\section{Methods and Techniques}

The architectural project roughly consists of the following five phases: a. conceptual design, b. design development, c. construction documents, d. bidding/permitting, and e. construction administration (Fig.5).

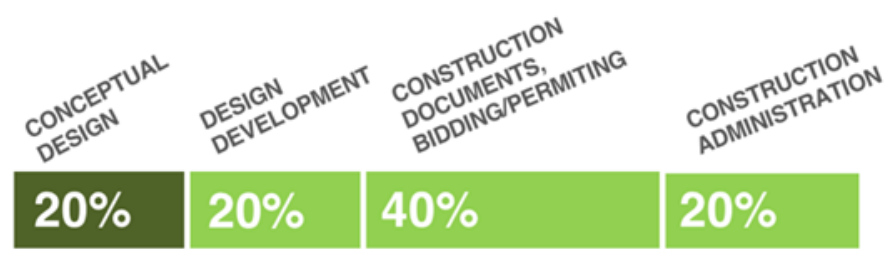

Fig. 5: The design phases of an architectural project

The platform simply facilitates currently only the conceptual design phase. The idea is that, for example, a client in Boston crowdsources the best design, say from a designer in Armenia, and then hands the design over to a local professional to implement and complete the later four phases of the project locally in Boston.

The crowdsourcing protocol is as follows: The client describes the project, uploads images of the project spaces, sets a timeline, and puts forth an award amount. The system suggests an award based on the complexity of the project, however, the client is free to overwrite the suggested amount and post any award above the base minimum. In the later part of this paper I will explain how we utilized artificial intelligence to recommend award amounts. Once the award is paid by the client, the project is automatically set online and notifications are sent out to designers. They can then review the project, sign-up and submit their work.

During the competition, designers and clients can communicate on a public project wall. The identity of designers and clients are kept anonymous, however the communication itself is open to the public, so everyone can benefit from the questions, comments, and answers posted on the wall. At the end of the deadline, the client reviews all submissions, and rates them through a rating module, ranks all entries, and -if wanted- shares all or a subset of submission with their family and friends. In addition, clients can post submissions for public voting, in which case any user on the system can vote on tem on a 1-10 scale. All ratings are viewable to the client at their analytics page. The client can then make their final decision and submit their winning designers to the system. The platform distributes the awards to the winning designers: The 1st designer gets $60 \%$ of the award, the 2nd designer gets $30 \%$ of the award, and the 3rd designer gets $10 \%$ of the award. Clients can also issue honorable mentions, which are not monetary awards, but will give designers additional points that helps them rank higher on the platform's designer charts.

\section{The Case Study}

As a case study I will present a project for a façade design we completed on the platform in 2016 . The client of the project was Forum8, a technology company out of Japan. Forum8 was looking to improve the appearance of their employees' residences in Shinagawa, Tokyo.

\subsection{The Brief}

The challenge was to design a façade for the northern elevation of an existing building, which currently houses employees of the company. There were few restrictions, for example, the design had to be offset from the sidewalk by one meter. Designers were asked to generate also the main entrance on this face of the building. Forum 8 challenged designers to "envision an aesthetic façade that appeals to the eyes of both residents and guests as they are welcomed into the building." [3] The building is a small-scale podium-tower building and is four stories high, with the first floor being the podium on which a short three-story tower rest (Fig.6). 


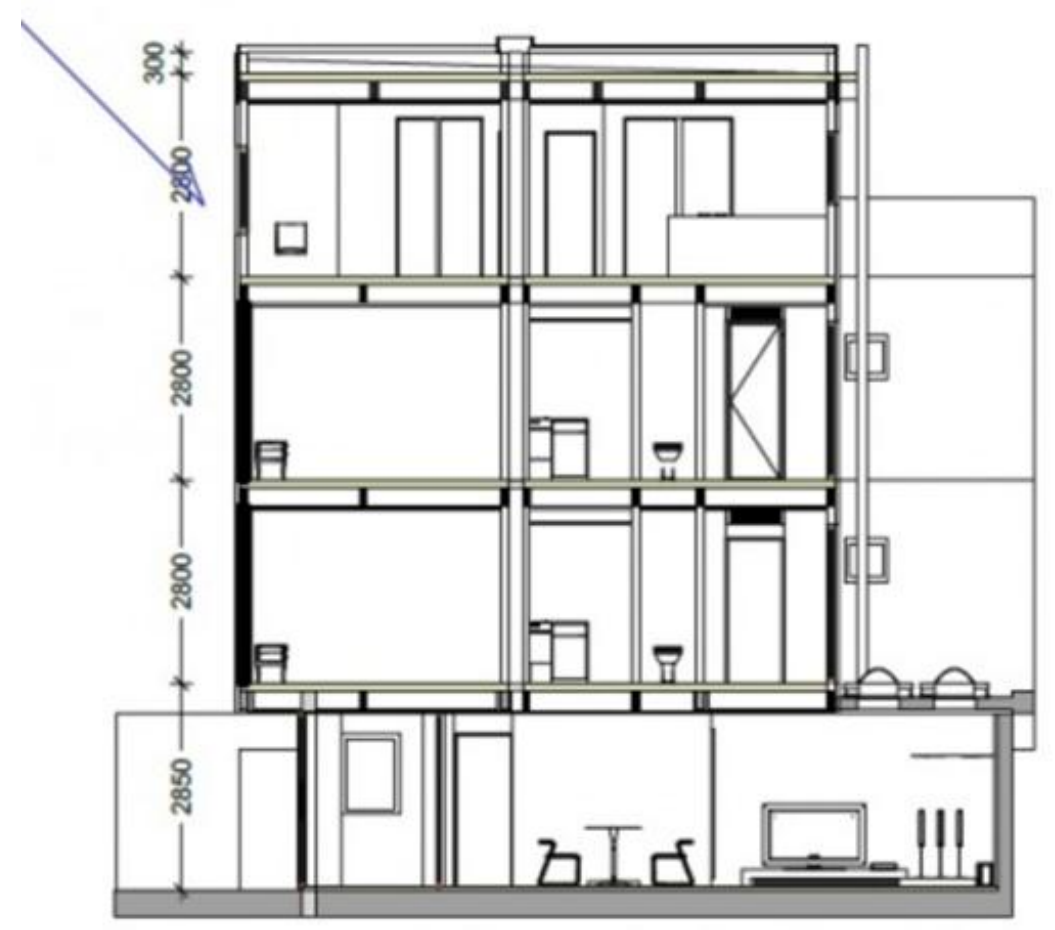

Fig. 6: Section through the existing Takanawa house, Tokyo.

The designers were given base drawings of the building and granted access to the Forum8 VR-Cloud software, which allowed them into explore their designs in a $3 \mathrm{D}$ virtual simulation of the real context. The deliverables were a 200-word description of the project, one elevation drawing, one perspective or axonometric drawing, and a digital 3D model. The award was set to $\$ 938$, with a timeline of nine days to complete and upload the designs.

\subsection{The Result}

During the course of the competition 50 designers signed up from various countries. The top five countries by the number of participation were the United States (9), Bosnia-Herzegovina (6), Romania (5), Canada (4), and Italy (4). Of these, $25 \%$ of designers were based in the Americas, $18.8 \%$ in Asia, $53.1 \%$ in Europe, and $3.2 \%$ in Africa $-65.6 \%$ of participants were male and $20.3 \%$ female. At the end of the nine days, 29 designers in total submitted their designs on the platform. The submissions were opened for public voting and received in total 1,055 votes: 810 came from regular users, 85 votes from design-experts, and 160 votes from family and friends of the client (Fig.7). 


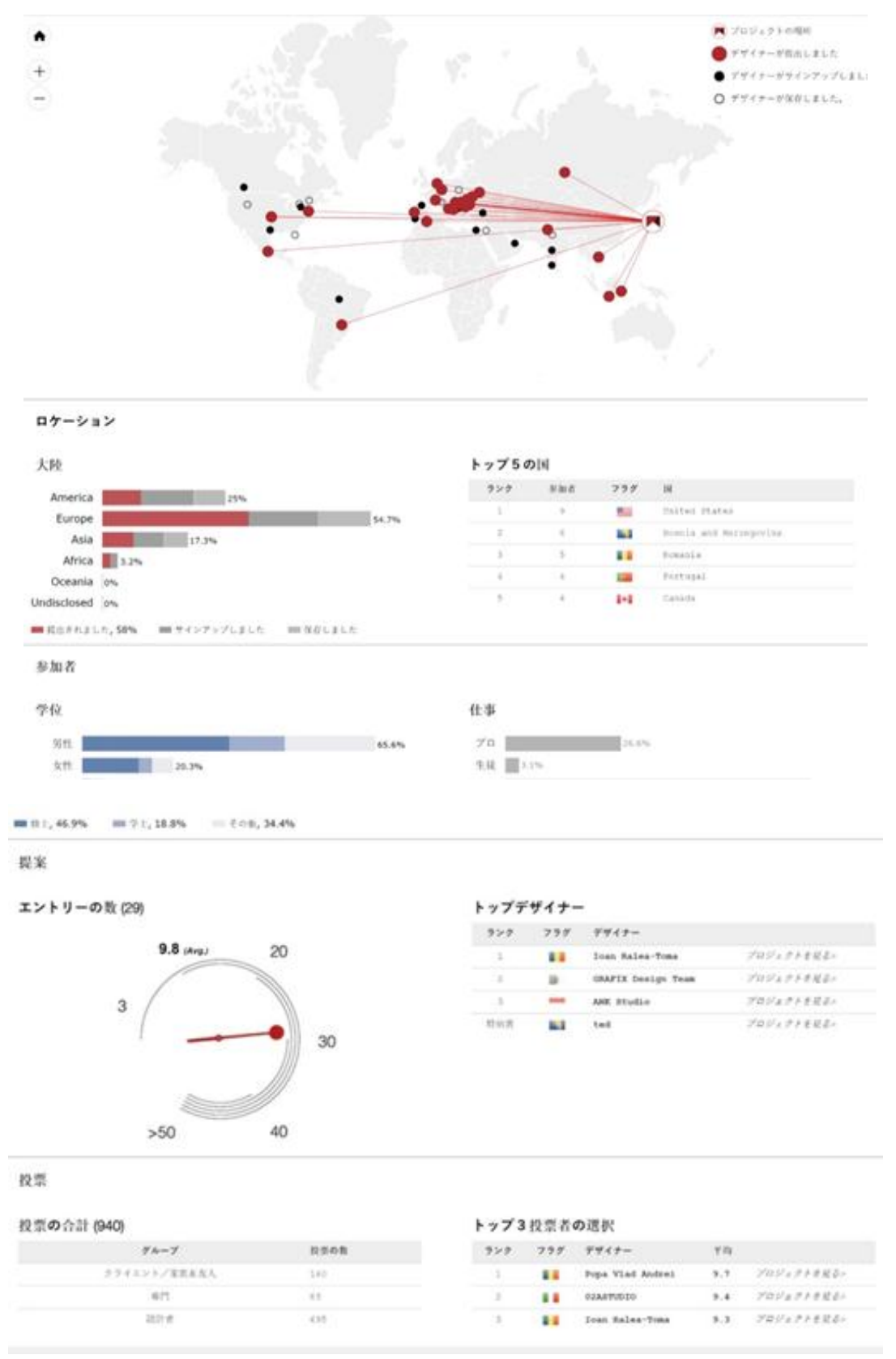

Fig. 7: Analytics page of the Takanawa House Façade Project, Tokyo.

Forum8 selected the design submitted by Popa Vlad Andrei of Romania, as the winning scheme (Fig.8). Popa's design received an average score of 9.7 by platform users. The designers described their projects in the following way:

"The base concept of this proposal is to reinterpret traditional Japanese facades in a contemporary way in order to create different instances of the same spaces that acknowledge one's privacy but is also open towards the outside. This is translated into an interactive façade that uses traditional materials such as wood as a threshold between two worlds - inside and outside. A subtle steel grid is the frame support for the fixed wood planks that covers up the entire northern façade, while profiled rails allow the residents to glide the wood panels at their own wish, for the desired effect. The wood, a warm material reminds the passerby of traditional woodwork crafts, but it also scales down the building to human size, helping the volume fit in a dense urban environment." [4] 

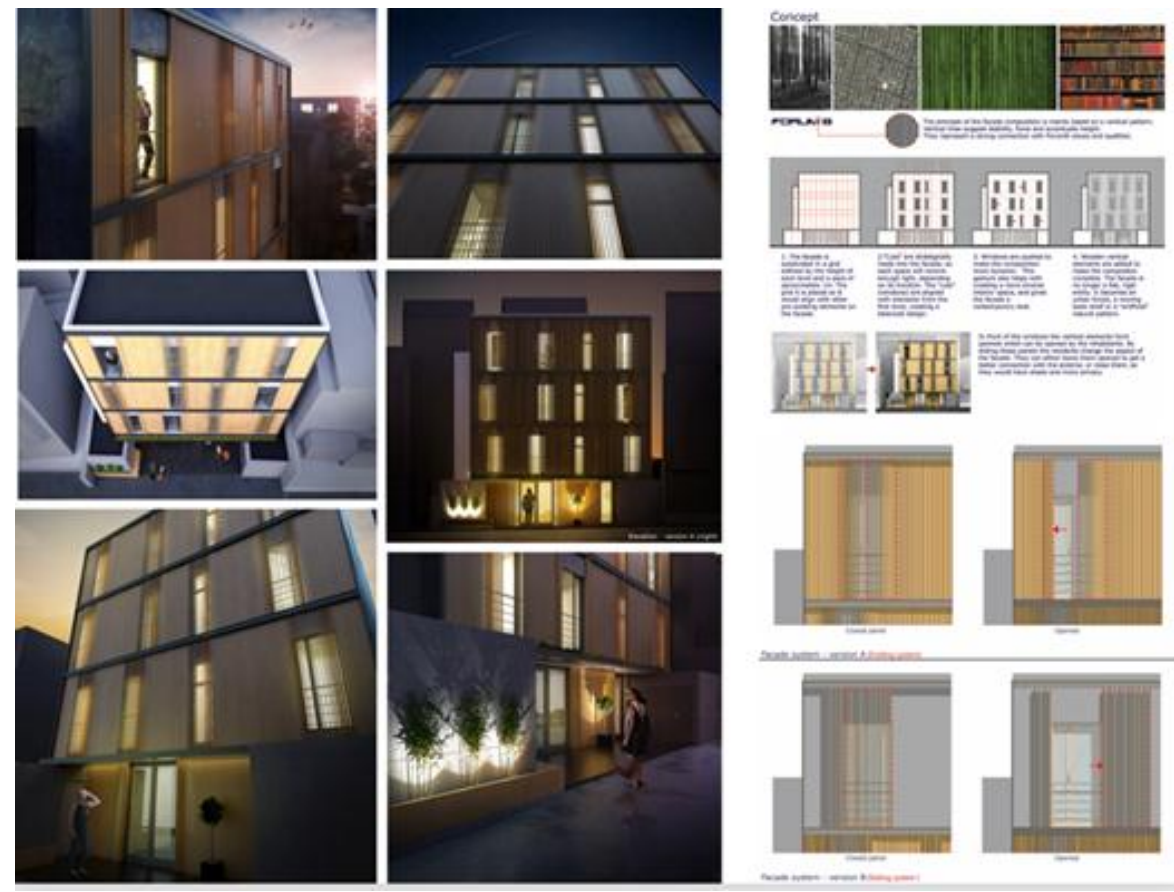

Fig. 8: Design entry by Popa Vlad Andrei

The second place went to the GRAFIX Design Team from Algeria. It received a score of 7.7, and the third place went to ANK Studio from Indonesia. Their submission scored 7.3 in public voting. Their main idea was a "green façade that consists of two parts: entrance level, using vertical cantilever planter boxes (either with hydroponic/ aeroponic system) where you can plant edible local herbs and vegetables; and upper apartment levels, using green screens with trellis system to grow climbing fruit vines, to reduce heat gain during summer and creating a privacy layer." [5]

Crowdsourcing the Takanawa house façade had significant benefits over traditional methods of design acquisition. First, the clients were able to use a merit-based delivery system and create an even playing field for designers; second, they received a multitude of design solutions; and third, they were able to consult expert designers through the voting procedure while picking their winning scheme.

\section{Future Work: Deep Learning}

The platform collected thousands of designs over the years. In 2017, we used this data and trained neural networks towards exploring three issues: a. to develop a recommendation system for award amounts. For example, when a client launches a project, the system looks into the performance of similar type completed projects on the platform based on 53 criteria, e.g. the number of submissions, sign-ups, timeline, awards, etc. and then makes an award suggestion at an optimum amount, i.e. award amount that yield high performing results; $b$. to generate function-driven designs. In other words, to train neural networks to evaluate designs based on function targets, and to discover latent high- performing building blocks that can be re-combined into new and unprecedented assemblies. Our goal is to eventually generate conceptual designs that could compete in realworld design projects on the platform (Fig.9). 


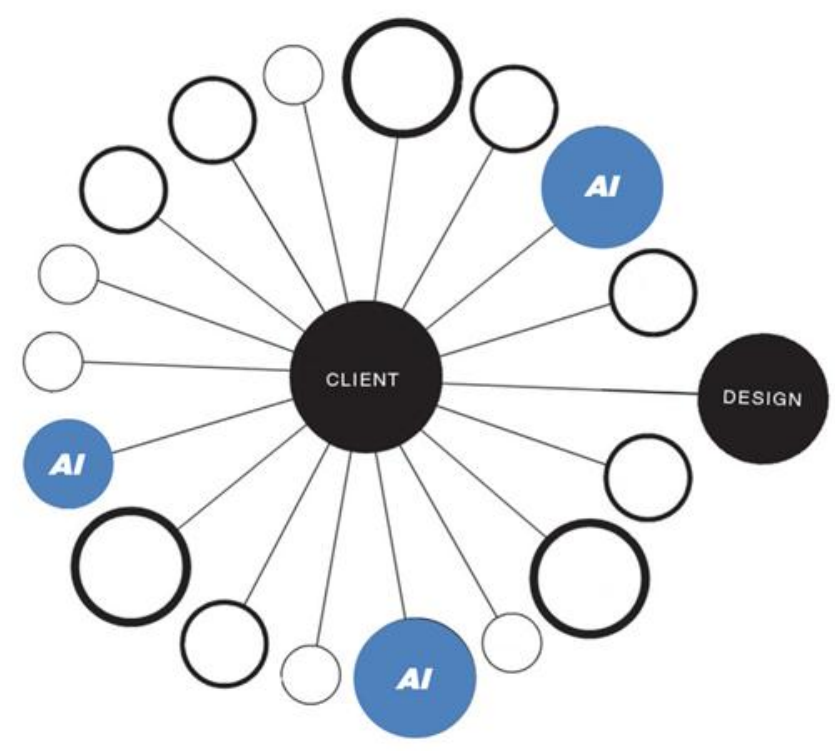

Fig 9: Crowdsourcing with human and AI generated projects.

\section{Conclusion}

In this paper, I presented crowdsourcing as an alternative method to traditional acquisition models, such as RFP. I further elaborated on the crowdsourcing protocol through the case study of the Takanawa House Façade project in Tokyo, Japan. The platform, gave us an opportunity to explore the potential of crowdsourcing as a new design delivery method. I argue that crowdsourcing can potentially create a level playing field for designers all around the globe and democratize the production of architectural spaces. In addition, I elaborated on our research on AI, and its potential to deliver conceptual designs. Undoubtedly, more research needs to be conducted in the field to unearth the full potential of crowdsourcing and AI systems in design generation.

\section{Acknowledgment}

I want to thank Forum8 for launching the crowdsourcing project on Arcbazar.com, and providing their VR Cloud software product for testing and exploration.

\section{References}

[1] I. As, T. Nagakura, "Architecture for the crowd by the crowd: A New Model for Design Acquisition," IJAUS International Journal of Architecture and Urban Studies, Vol1, Issue 2, pp.68-76, Fall 2016.

[2] Global Architectural Services - Global Market Research Report, IbisWorld, 2017, Available: https://www.ibisworld.com/industry-trends/global-industry-reports/business-activities/architectural-services.html

[3] Takanawa House Façade Design, Arcbazar.com, 2016, Available: https://www.arcbazar.com/facadedesign/competition/takanawa-house-facade-design-japan-tokyo

[4] Andrei, Popa Vlad, Takanawa House Façade Design, Arcbazar.com, 2016, Available: https://www.arcbazar.com/facade-design/project/popa-vlad-andrei-takanawa-house-facade-design-japan-tokyo

[5] ANK Studio, Takanawa House Façade Design, Arcbazar.com, 2016, Available: https://www.arcbazar.com/facadedesign/project/ank-studio-takanawa-house-facade-design-japan-tokyo 